\title{
Vaccination in Old Age
}

With increasing age the immune system undergoes characteristic changes, termed immunosenescence. Cells of the innate immune system - such as neutrophilic granulocytes, monocytes, macrophages and dendritic cells - show decreased migration, signaling, phagocytosis and antimicrobial activity. As a consequence, the first line of defence against infections is impaired and pathogens are less efficiently eliminated. Another important task of the innate immune cells is antigen processing and presentation. After phagocytosis pathogenderived proteins are fragmented into peptides, which are then loaded onto MHC (major histocompatibility complex) proteins and presented on the cell surface. These complexes are recognized by $T$ cells and induce their activation. Antigen processing and presentation is impaired in old age leading to a diminished activation of $\mathrm{T}$ cells. The output of naïve T and B cells is decreased, whereas antigen-experienced and particularly highly differentiated lymphocytes accumulate. As a consequence, the repertoire and diversity of the adaptive immune system is diminished. Cellular functions of T cells, such as signaling after antigenic stimulation, proliferation and cytokine production are impaired limiting T cell responses. In addition, somatic hypermutation and class switch, which take place in $B$ cells to optimize antibody production, are diminished in older age. T cell help is required for these processes and the age-related impairments we observe are caused by intrinsic defects of the B cells as well as by suboptimal $T$ cell help. Overall, these age-related changes of the immune system contribute to increased incidence and severity of infections in old age. Infectious diseases are frequently associated with long-term sequelae such as impairments in activities of daily living, onset of frailty, or the loss of independence. In view of demographic changes leading to a dramatic increase of old and very old individuals, this represents a serious challenge for public health systems. The prevention of infectious diseases is therefore an important measure to ensure healthy aging and improve the quality of life.

Vaccination is the most effective measure to achieve this goal and vaccination rec- ommendations in most countries include specific guidelines for the older population. Vaccination against Streptococcus pneumoniae and influenza is usually recommended for persons with underlying diseases and for the older population with heterogeneous age limits between $\geq 50$ years and $\geq 65$ years. Some countries also recommend vaccination against herpes zoster. Several vaccines are recommended for all adults, such as regular booster shots against tetanus/diphtheria/pertussis/polio, or for specific groups, e.g. vaccination against tick-borne encephalitis in endemic areas or travel vaccines. These are also relevant for older individuals. Most currently used vaccines are less immunogenic and effective in older compared to younger adults. Induction of immune responses is frequently also slower in older persons. This can be of particular relevance for travel vaccines, which should be given early enough before starting the journey. Immunogenicity of influenza vaccines is usually measured by hemagglutination inhibition assay (HAI), which quantifies antibodies specific for the viral hemagglutinin. Many studies demonstrated that antibody concentrations after vaccination are lower in older compared to younger adults and that co-morbidities and frailty further decrease responsiveness to vaccination. Several strategies including higher antigen dose, alternative routes of administration, and the use of adjuvants have been implemented to improve immunogenicity of influenza vaccines and lead to moderately higher antibody concentrations. Research on universal vaccines against influenza is ongoing in order to overcome the limitations of the current strain-specific vaccines.

Invasive pneumococcal disease (bacteremia, meningitis etc.) mainly affects young children and older adults. S. pneumoniae is also a common cause of community-acquired pneumonia (CAP) in the elderly. A 23-valent polysaccharide vaccine has been used for many years for older adults, but polysaccharides induce lgM-dominated antibody responses without adequate immunological memory, as they are T cell-independent antigens. Conjugated vaccines, which contain polysaccharides coupled to carrier proteins are able to overcome these limitations to a certain extent, as these antigens are processed by the immune system in a $\mathrm{T}$ cell dependent way. After the very successful use of a 7-valent conjugate vaccine for children, the next generation 13-valent conjugate vaccine has been introduced for children and older adults. In a large randomized trial in persons over 65 years of age, $45.6 \%$ fewer first episodes of vaccine-type CAP requiring hospitalization and $75.0 \%$ fewer first episodes of vaccinetype invasive pneumococcal disease occurred in the vaccine group compared to placebo. Vaccination recommendations for S. pneumoniae are heterogeneous. Some countries still recommend the polysaccharide vaccine, while others recommend the conjugate vaccine alone or followed by the polysaccharide vaccine at least one year later.

Almost all adults are infected with varicella zoster virus, which establishes life-long latency after primary infection (chickenpox). Episodes of viral reactivation occur throughout life, but are usually controlled by adaptive immune responses. In the absence of sufficient immunological control, e.g. due to immunosenescence or immunosuppression, reactivation of varicella zoster virus leads to herpes zoster (shingles). Acute episodes of herpes zoster are frequently followed by post-herpetic neuralgia (PHN), characterized by long-lasting severe pain after the resolution of the zoster rash. A live-attenuated vaccine against herpes zoster, which boosts the natural immune response developed after primary infection, has been used for several years. It was shown to reduce the incidence of herpes zoster by $51 \%$ and the incidence of PHN by $67 \%$ compared to placebo in a randomized trial including persons older than 60 years. The protective effect of the vaccine was lower in the very old, and longterm follow-up studies showed that protection waned over time. Recently, an inactivated vaccine against herpes zoster containing the viral glycoprotein $\mathrm{gE}$ and the adjuvant $\mathrm{ASO1}_{\mathrm{B}}$ has been licensed. In randomized clinical trials efficacy of this vaccine has been shown to be $>90 \%$ and did not decrease with age in the target population (> 50 years). This vaccine can 
also be used in immunosuppressed patients, which are at high risk for herpes zoster, but cannot be vaccinated with live vaccines.

Novel vaccines against various other pathogens are currently studied and developed by many academic and commercial organisations. Respiratory syncytial virus causes significant morbidity in the older population, and several vaccines candidates are currently in development. Bacterial infections, particularly nosocomial infections, are frequent in the elderly and vaccines against these pathogens have the potential to prevent disease as well as reduce the use of antibiotics. In view of increasing antibiotic resistance, vaccines against these pathogens are highly desirable.

In addition to novel vaccine developments, it is crucial to increase awareness for the importance of vaccination beyond the pediatric setting, as vaccination coverage is still far from optimal for the older population.

\section{Conflict of Interest}

Presentations at scientific meetings and symposiums upon invitation by Novartis, GSK, Sanofi Pasteur, Merck Vaccines, MSD, Pfizer. Invitation to events by the Austrian Association of Vaccine Manufacturers. Advisor for Novartis, LimmaTech Biologicals AG, Novavax Inc., Janssen LLC
Autorinnen/Autoren

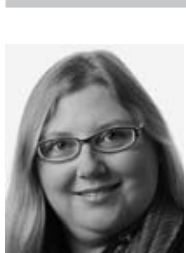

\section{Birgit Weinberger}

PD Dr., Institut für Biomedizinische Alternsforschung, Universität Innsbruck, Österreich

\section{Correspondence}

PD Dr. Birgit Weinberger

Institut für Biomedizinische

Alternsforschung

Universität Innsbruck

Rennweg 10

6020 Innsbruck

Österreich

birgit.weinberger@uibk.ac.at 